\title{
Exercício físico e envelhecimento: benefícios à saúde e características de programas desenvolvidos pelo LABSAU/IEFD/UERJ
}

\author{
Exercise and aging: health benefits and characteristics of the intervention \\ programs at LABSAU/IEFD/UERJ
}

\section{Ejercicio físico y envejecimiento: beneficios para la salud y características de los programas desarrollados por el LABSAU/IEF/UERJ}

\author{
Nádia L. Silva*, Cristiane Brasil, Hélio Furtado, João Costa, Paulo Farinatti
}

\section{Resumo}

Progressos sociais, políticos, econômicos e ambientais e o avanço científico em diferentes áreas têm proporcionado melhorias nas condições de vida e saúde das populações, desse modo influenciando a expectativa de vida. Analisado sob a ótica das teorias biológicas, o envelhecimento é caracterizado pela degeneração das funções e estruturas orgânicas. Sendo assim, tende a haver um declínio gradativo das capacidades motoras. Nesse contexto, o exercício físico regular vem sendo apontado como um fator importante no que se refere à preservação da autonomia funcional e por auxiliar na prevenção e no controle de doenças crônico-degenerativas. O presente artigo tem por objetivo revisar a literatura sobre os benefícios do exercício físico para a saúde e qualidade de vida do idoso, bem como apresentar para essa população propostas de programas de exercícios físicos que vêm sendo desenvolvidos no Laboratório de Atividade Física e Promoção da Saúde do Instituto de Educação Física e Desportos da Universidade do Estado do Rio de Janeiro (LABSAU). Em suma, o exercício físico sistematizado pode acarretar benefícios na esfera física e mental, proporcionando melhor qualidade de vida ao idoso. O LABSAU, por meio de seus projetos e parcerias, vem oferecendo oportunidades de engajamento da pessoa idosa em programas regulares de atividades físicas de diversas naturezas, contribuindo com o desenvolvimento dessa área de interesse no estado do Rio de Janeiro.

Descritores: Exercício físico; Idoso; Aptidão física; Qualidade de vida; Educação física e treinamento.

\begin{abstract}
Social, political, economic and environmental progress and scientific development in different areas improved overall health and quality of life, therefore increasing life expectancy. In a biological perspective, aging is defined as the degeneration of organic functions and structures, with consequent decline of motor and mental capacities. In this context, regular physical exercise has been considered an important strategy to preserve functional autonomy and to prevent chronic-degenerative diseases. This article aimed at reviewing the literature on the potential benefits of physical exercise to health and quality of life in the elderly, as well as to describe exercise intervention programs for this population developed by the Laboratory of Physical Activity and Health Promotion of the Institute of Physical Education and Sports from the University of Rio de Janeiro State (LABSAU). It can be concluded that systematic exercise can bring about several benefits in both physical and mental health, improving the quality of life in the old age. Through its intervention programs and partnerships, LABSAU has been offering opportunities of engagement of the elderly in physical activity programs of different natures, thus contributing to the development of this area of interest in the state of Rio de Janeiro.
\end{abstract}

Keywords: Exercise; Aged; Physical fitness; Quality of life; Physical education and training. 


\section{Resumen}

Los progresos sociales, políticos, económicos, ambientales y el avance científico en diferentes áreas han proporcionado mejoras en las condiciones de vida y salud de las poblaciones, influyendo así en la esperanza de vida. Analizado bajo la óptica de las teorías biológicas, el envejecimiento se caracteriza por la degeneración de las funciones y las estructuras orgánicas. Por lo tanto, tiende a haber un declive gradual de las capacidades motoras. En este contexto, el ejercicio físico regular viene siendo indicado como un factor importante con respecto a la preservación de la autonomía funcional y como apoyo en la prevención y control de enfermedades crónico-degenerativas. El presente artículo tiene por objetivo revisar la literatura sobre los beneficios del ejercicio físico para la salud y la calidad de vida de las personas mayores, así como presentar propuestas de programas de ejercicios físicos para esta población, que vienen siendo desarrollados en el Laboratorio de Actividad Física y Promoción de la Salud del Instituto de Educación Física y Deportes de la Universidad del Estado de Rio de Janeiro (LABSAU). En resumen, el ejercicio físico sistematizado puede producir beneficios en la esfera física y mental, proporcionando mejor calidad de vida al adulto mayor. El LABSAU, a través de sus proyectos y asociaciones, viene ofreciendo oportunidades para la participación de los adultos mayores en programas regulares de actividades físicas de diversas naturalezas, contribuyendo al desarrollo de esta área de interés en el estado de Río de Janeiro.

Palabras clave: Ejercicios físicos; Adulto mayor; Aptitud física; Calidad de vida; Educación y entrenamiento físico.

\section{Introdução}

Os progressos sociais, políticos, econômicos e ambientais, bem como o avanço científico em diferentes áreas, têm proporcionado de forma contínua e sustentada melhorias nas condições de vida e saúde das populações da grande maioria dos países, influenciando desse modo a expectativa de vida. ${ }^{1}$

Segundo dados da Organização Mundial da Saúde, entre 1950 e 1998, a representatividade populacional de pessoas com idade maior ou igual 60 anos no mundo aumentou de oito para $10 \%{ }^{2}$ A projeção realizada para 2050 estima que as pessoas dessa faixa etária representem $20 \%$ da população mundial, aproximadamente 2 bilhões de habitantes. Destes, mais de $60 \%$ estarão vivendo em países em desenvolvimento. ${ }^{2}$ No Brasil, o número de idosos passou de 3 milhões em 1960, para 7 milhões em 1975, e 21 milhões em 2010. Além disso, vale ressaltar que, nesse contingente, a população "muito idosa", ou seja, com mais de 80 anos, é aquela cujas taxas de crescimento são mais expressivas. $^{3}$

Envelhecer é um processo que se dá paulatinamente e sofre interferências de variáveis biológicas e sociais. ${ }^{4}$ Analisado sob a ótica das teorias biológicas, o envelhecimento é caracterizado pela degeneração das funções e estruturas orgânicas. Desse modo, tende a haver um declínio gradativo das capacidades motoras, como a redução da flexibilidade, velocidade, capacidade aeróbia e força muscular. ${ }^{5-7}$ Em última análise, a realização das atividades diárias e, por conseguinte, a manutenção de um estilo de vida ativo vão se tornando mais difíceis. ${ }^{8}$

Aos efeitos típicos do processo de envelhecimento, soma-se a perda gradual da aptidão física funcional. Nesse contexto, o oferecimento de oportunidades de engajamento em programas de exercício físico regular é apontado como fator importante, no que se refere à preservação da autonomia funcional ${ }^{9} \mathrm{e}$ por auxiliar na prevenção e controle de muitas doenças crônico-degenerativas. ${ }^{10}$ Assim sendo, o presente artigo tem por objetivo descrever os benefícios do exercício físico para a saúde e qualidade de vida do idoso, bem como apresentar para essa população propostas de programas de exercícios físicos que vêm sendo desenvolvidos no Laboratório de Atividade Física e Promoção da Saúde do Instituto de Educação Física e Desportos da Universidade do Estado do Rio de Janeiro (LABSAU/IEFD/UERJ).

\section{Benefícios dos exercícios físicos}

\section{para os idosos}

Em qualquer faixa etária, inclusive as mais avançadas, manter uma rotina diária de exercícios físicos pode trazer benefícios biológicos e psicossociais. Frequentar um programa regular de exercícios físicos, como ginástica, hidroginástica, musculação, ou qualquer outra modalidade, pode ser muito mais benéfico do que parece. Para maior clareza, esses benefícios serão descritos em subseções. 


\section{Benefícios biológicos}

Idosos que praticam atividade física regularmente, em geral, mantêm o corpo em boas condições físicas. Alguns pesquisadores utilizam como estratégia de avaliação da "idade biológica" a análise de vários indicadores que estabelecem o quanto está sendo eficiente o funcionamento do corpo." $\mathrm{O}$ estilo de vida do indivíduo interfere sobremaneira nesses indicadores, como a manutenção de uma alimentação equilibrada ao longo da vida, prática regular de exercícios físicos, horas de sono adequadas, dentre outros fatores.,112

Paffenbarger e colaboradores ${ }^{12}$ publicaram estudo clássico relacionando exercício físico e mortalidade. Durante 22 anos, 14.000 ex-alunos de Harvard foram acompanhados, verificando-se que os indivíduos que pararam de praticar esportes tiveram $35 \%$ de aumento no risco de morte em comparação com os que continuaram ativos. Porém, aqueles que começaram a praticar esportes tiveram índice $21 \%$ menor de mortalidade que aqueles habitualmente sedentários. O aumento na expectativa de vida quando os mais ativos foram comparados aos pouco ativos foi em média de 2,51 anos para indivíduos de 35-39 anos de idade no início do estudo e de 0,42 anos nos indivíduos de 75-79 anos. A pesquisa demonstrou, ainda, que a porcentagem de indivíduos acima de 80 anos foi maior entre indivíduos mais ativos $(69,7 \%)$ do que nos menos ativos (59,8\%).

De fato, indivíduos que mantêm prática regular de exercícios ao longo da vida parecem ter expectativa de vida maior do que a média da população. Isso porque essa prática pode retardar o período em que a capacidade funcional declina, ou seja, aumenta a probabilidade de se manter a independência funcional por mais tempo, evitando com isso a necessidade de cuidados por terceiros para o desempenho de atividades diárias (alimentação, higiene pessoal, vestir-se, movimentar-se, fazer compras, tomar medicamentos etc). ${ }^{13}$

Além do impacto sobre a independência funcional e consequente aumento da expectativa de vida, a prática regular de exercícios físicos guarda relações importantes com a prevenção e o controle de muitas doenças crônico-degenerativas, dentre as quais se pode citar as doenças cardiovasculares, diabetes tipo II, câncer, osteoporose e a sarcopenia. ${ }^{14}$
Para que os exercícios físicos possam contribuir de fato para o envelhecimento com mais qualidade, entidades normativas como o American College of Sports Medicine (ACSM) $)^{15}$ recomendam que tanto exercícios aeróbios quanto de força sejam realizados, uma vez que cada tipo de exercício trará benefícios biológicos diferenciados. Sendo assim, as seções a seguir discorrem sobre os benefícios desses dois tipos de exercícios.

\section{Benefícios dos exercícios aeróbios}

Consideram-se como exercícios aeróbios aqueles que utilizam grandes grupos musculares, realizados de forma contínua por um período de tempo superior a três minutos e que utilizam predominantemente o sistema oxidativo de oferta de energia para a sua prática: caminhar, correr, pedalar, nadar, dentre outros. Segundo o ACSM, ${ }^{15}$ para que o idoso atinja os objetivos provenientes de sua prática, esses exercícios devem ser realizados com intensidade de moderada a vigorosa, acumulando pelo menos de 30 a 60 minutos diários, com uma frequência de pelo menos três a cinco dias por semana.

Durante o envelhecimento, acontece uma redução do volume de oxigênio absorvido, aproximadamente $10 \%$ por década a partir dos 30 anos, de forma que aos 60 anos um idoso sedentário pode ter aproximadamente $60 \%$ da capacidade aeróbia que tinha aos 25 anos. $^{16}$ Pesquisas comprovam que o volume de oxigênio absorvido de indivíduos treinados com mais de 50 anos é de 20 a 30\% maior que a de indivíduos sedentários da mesma idade. ${ }^{17} \mathrm{O} \mathrm{VO}_{2}$ máx (consumo máximo de oxigênio), principal indicador de aptidão física, declina aproximadamente $1 \%$ a cada ano em homens e mulheres após os 25 anos, sendo que os fisicamente ativos preservam uma capacidade aeróbia mais alta que os sedentários da mesma idade, mesmo em idades avançadas. ${ }^{16}$

O exercício aeróbio regular induz alterações favoráveis também no peso e composição corporal. Observa-se uma redução da massa de gordura, peso corporal e gordura intra-abdominal em idosos que participam de programas de exercícios prolongados. ${ }^{18} \mathrm{O}$ fator mais importante em programas que buscam a redução de peso é o gasto energético total. ${ }^{18} \mathrm{O}$ exercício aeróbio pode diminuir a gordura corporal por meio do 
aumento do gasto energético diário $(500 \mathrm{~kJ} /$ dia). Esse exercício pode ser mais eficiente se acompanhado de um pequeno declínio na dieta alimentar (igualmente de $500 \mathrm{~kJ} / \mathrm{dia}$ ). ${ }^{19}$ Ao se realizar um exercício aeróbio contínuo, utilizam-se grandes grupos musculares, produzindo um gasto energético de moderado a alto. Esses exercícios são ideais para promover a perda de peso, consomem uma quantidade significativa de calorias, estimulam o metabolismo lipídico e reduzem a gordura corporal. ${ }^{18}$ A redução do peso corporal não está estreitamente relacionada à modalidade do exercício a ser praticado, mas sim à frequência, duração e intensidade com que é executado. ${ }^{20}$

Com o passar dos anos, o coração também não consegue mais manter o número de batimentos elevados como na juventude, isto é, a frequência cardíaca máxima (FC máx) diminui com a idade. ${ }^{13}$ No entanto, pesquisas comprovam que, entre indivíduos da mesma idade, os fisicamente ativos apresentam valores de FC máx maiores que os sedentários, sugerindo que o exercício produz um funcionamento mais eficiente de todo o sistema circulatório. ${ }^{21}$ Outro aspecto não menos relevante dos efeitos dos exercícios aeróbios sobre o sistema circulatório diz respeito à pressão arterial (PA). Com o passar da idade, há uma tendência de aumento da PA e a hipertensão passa a ser uma doença muito comum nos idosos. ${ }^{22}$ Vários estudos sugerem a prática de exercícios físicos como forma de tratamento e controle da hipertensão arterial, demonstrando que uma única sessão de exercícios físicos pode gerar decréscimo temporário da PA, fenômeno denominado hipotensão pós-exercício (HPE). Esse efeito, reproduzido sistemática e cotidianamente, pode levar à diminuição crônica da PA. ${ }^{23}$

\section{Benefícios dos exercícios de força muscular}

Os exercícios de força muscular são aqueles realizados contra uma resistência. Os mais comuns envolvem mobilização de cargas (musculação), mas outras formas de resistência podem proporcionar a manutenção da força muscular. Pessoas idosas têm grande necessidade de manter níveis razoáveis de força muscular, já que essa capacidade física diminui com o processo de envelhecimento. A força muscular apresenta um declínio, apesar de lento, já a partir da quarta década de vida, acentuando-se gradativamente após os 50 anos de idade. ${ }^{24}$

A perda de força tem impacto sobre os níveis de independência funcional nas idades mais avançadas. Latham e colaboradores, ${ }^{25}$ por exemplo, relataram que níveis reduzidos de força estariam associados a uma menor velocidade de caminhada e aumento do risco de quedas e fraturas nas pessoas mais velhas. Além disso, níveis reduzidos de força levam à perda progressiva de aspectos relacionados à autonomia funcional, com limitações importantes da capacidade para a realização das tarefas do dia a dia. ${ }^{24}$

Durante o envelhecimento, o declínio da força muscular com a idade decorre da diminuição e substituição do tecido muscular por gordura e tecido conjuntivo. ${ }^{26} \mathrm{~A}$ sarcopenia, como é chamado esse processo, caracteriza-se principalmente pela diminuição da quantidade e capacidade das proteínas contráteis em exercerem tensão suficiente para vencer uma resistência na realização de uma tarefa. ${ }^{26}$ Para Hurley e colaboradores, essa involução da massa muscular não tem implicação somente na força, mas também sobre as funções metabólicas e cardiovasculares. ${ }^{27}$ Em longo prazo, isso pode tornar os idosos mais suscetíveis ao desenvolvimento de condições patológicas como obesidade e diabetes. ${ }^{28}$

No âmbito dos exercícios físicos, há evidências de que o treinamento de força induz a melhorias na função e estrutura muscular, articular e óssea, ${ }^{25}$ bem como metabólica e cardiovascular, ${ }^{29}$ favorecendo aspectos importantes para a saúde e qualidade da vida dos idosos, por exemplo a manutenção da aptidão funcional ${ }^{9} \mathrm{e} o$ controle de peso corporal. ${ }^{29}$ Achados como esses levaram à aceitação de que é importante incluir programas de treinamento da força muscular para a conservação da capacidade de trabalho e autonomia do idoso. A ideia de que a prática de exercícios resistidos seja incluída no planejamento de programas de atividades físicas voltadas para a promoção da saúde de indivíduos de qualquer faixa etária e condições clínicas, ${ }^{30}$ desde que o treinamento seja adequadamente prescrito, é hoje praticamente consensual.

Outra importante pesquisa foi realizada por Fiatarone e colaboradores, ${ }^{31}$ demonstrando a eficiência do treinamento de força de alta inten- 
sidade em idosos com 86 a 96 anos de idade. O programa foi realizado três vezes por semana e teve a duração de oito semanas. O resultado apresentou um aumento de $174 \%$ na força muscular, caracterizando que a idade avançada não limita os benefícios que o treinamento de força pode oferecer. Pode-se observar, então, que a capacidade de os músculos responderem e se adaptarem aos estímulos fornecidos pelo treinamento mantém-se preservada em idosos, sendo o treinamento de força uma proposta rápida e eficiente de melhorar a sua rotina diária. ${ }^{16}$

\section{Benefícios psicossociais}

Idosos levam, em geral, a vida de forma muito solitária, tendo pouca oportunidade de relacionamento. Os programas de exercícios físicos em grupo oferecem um meio importante de atender à necessidade de um maior número de contato social. Ao serem praticados regularmente, podem favorecer uma mudança comportamental, que poderá proporcionar transformações emocionais e psicológicas, visto que o idoso quando se sente valorizado pode desencadear alterações positivas junto à sua família e meio social. ${ }^{13}$

Mantendo-se ativo, o idoso pode estimular a convivência social de várias formas: por meio do diálogo, trocando experiências e fazendo novas amizades. Ao praticar exercícios físicos de forma coletiva, minimiza-se o isolamento social e, acima de tudo, cria-se um convívio saudável. Esse aspecto tem uma relação direta com a saúde psicológica do idoso. Pesquisas demonstraram que os exercícios físicos têm grande influência sobre o autoconceito e o bem-estar emocional de indivíduos idosos e que a associação entre exercícios físicos e saúde psicológica apresenta-se particularmente forte entre as mulheres. ${ }^{19}$

Embora possamos encontrar possíveis respostas para efeitos positivos da prática regular de exercícios físicos sobre a depressão, a ansiedade e o humor no aumento da liberação de hormônios que agiriam na modulação emocional dos seres humanos, ${ }^{32}$ fazendo com que este fenômeno possa ser analisado como mais um benefício biológico do exercício físico, entende-se nesse estudo que distúrbios dessa natureza influenciam diretamente questões como o isolamento social, a autoestima e a qualidade de vida dos idosos, ${ }^{33}$ o que nos leva a tratar o tema sob a ótica psicossocial.
De fato, estudos prévios indicam que a prática sistemática do exercício físico para a população em geral está associada à ausência ou a poucos sintomas depressivos ou de ansiedade, transtornos muito comuns em idosos que podem levar ao isolamento e doenças mentais. ${ }^{34}$ Mesmo em indivíduos diagnosticados clinicamente como depressivos, o exercício físico tem se mostrado eficaz na redução dos sintomas associados à doença. ${ }^{34}$ Assim sendo, a prática regular de exercícios físicos deve ser considerada como uma alternativa não farmacológica de tratamento do transtorno depressivo, por representar baixo custo econômico, ser acessível e prevenir o declínio funcional do idoso. Além disso, o exercício físico apresenta contribuições importantes, sobretudo quando associado ao tratamento psicofarmacológico da depressão, auxiliando na recuperação da autoestima e autoconfiança. ${ }^{35}$

Para a Organização Mundial de Saúde (OMS), a participação em atividades físicas leves e moderadas pode retardar os declínios funcionais. Isto é, uma vida ativa melhora a saúde mental e contribui no gerenciamento de desordens como depressão e demência. Existem evidências de que idosos fisicamente ativos apresentam menor prevalência de doenças mentais do que os não ativos. ${ }^{2}$

Estilos de vida fisicamente ativos parecem trazer benefícios também à memória de idosos saudáveis ou portadores de condições patológicas que acometem o sistema cognitivo. ${ }^{36}$ Pesquisas demonstram que esses benefícios podem estar relacionados aos efeitos do exercício físico sobre o sistema cardiovascular ou mesmo a fatores

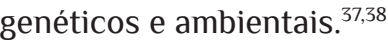

Outro aspecto importante nesse contexto é o estresse. Atualmente, grande parte da população convive com este fator em sua rotina diária. No cotidiano, muitas pessoas sofrem por terem seus direitos desrespeitados, transportes públicos ineficientes, aumento das exigências no trabalho e, na aposentadoria, muitas vezes, rendimentos reduzidos. ${ }^{13}$

De um modo geral, sugere-se que a prática regular de exercícios físicos poderia influenciar na melhora do humor, na diminuição da ansiedade e da depressão, o que pode também levar à diminuição do estresse. ${ }^{34}$ Idosos ativos, ao se exercitarem na intensidade adequada, liberam um hormônio chamado endorfina, ligado à sensação 
de bem-estar. A constância desse processo pode vir a modificar atitudes, aumentando a autoconfiança por meio da superação proporcionada pelo treinamento periódico. ${ }^{20}$

Em suma, buscando alternativas para que os idosos participem de programas de exercícios regulares, aumentam-se as chances de lhes proporcionar maior estabilidade emocional, estímulo à memória, valorização da imagem positiva do seu corpo e sensação de bem-estar, diminuindo-se a depressão e ansiedade. ${ }^{34}$

\section{Programas de exercícios físicos que vêm sendo desenvolvidos para idosos no LABSAU (IEFD/UERJ)}

O LABSAU vem desenvolvendo projetos de ensino, pesquisa e extensão na perspectiva de contribuir para a oferta de exercícios físicos regulares e seguros para indivíduos idosos, para formar recursos humanos qualificados à condução de aulas para essa população e gerar conhecimento sobre as relações entre atividade física e envelhecimento. A presente seção apresenta, ainda que brevemente, as características dos programas de exercícios físicos ofertados pelo laboratório.

\section{Idosos em Movimento: Mantendo a}

\section{Autonomia (IMMA)}

O projeto "Idosos em Movimento: Mantendo a Autonomia" (IMMA) foi criado em 17 de outubro de 1989, para atender pessoas com mais de 60 anos de idade e oferecer uma rotina de exercícios físicos de forma segura, especializada e embasada teoricamente. Buscava-se uma contraposição ao quadro da época, em que se percebia um aumento da oferta de programas de exercícios físicos para essa faixa etária, mas sem nenhuma fundamentação científica, colocando em risco a saúde dos praticantes.

O projeto IMMA tem concentrado suas preocupações no aprimoramento dos aspectos metodológicos do ensino de atividades físicas para idosos, dentro de um contexto multicultural. Neste sentido, seus objetivos são: a) contribuir para a redução do isolamento dos participantes, induzido pela aposentadoria e pela diminuição da aptidão funcional; b) proporcionar o gosto pelo movimento, de modo que se torne uma estratégia para a manutenção da autonomia de ação dos indivíduos; c) integrar os idosos em uma atividade de grupo, de forma a fazê-los reencontrar, em contato com outros de idade semelhante, o prazer de se comunicarem e se expressarem; d) contribuir para a formação de recursos humanos para a condução de atividades físicas com indivíduos idosos; e) contribuir para a pesquisa e produção de conhecimentos no âmbito da atividade física e a autonomia de idosos.

As atividades desenvolvidas pelo projeto IMMA priorizam a segurança durante as práticas, incluindo os seguintes procedimentos: a) 'avaliação ampla do idoso'; b) aferição da pressão arterial de todos os idosos antes de cada aula; c) dosagem de glicose dos idosos portadores de diabetes, antes e após a aula; d) exibição de todas as rotinas preventivas na lista de chamada; e) procedimentos ligados a cuidados com o ambiente físico e o material para as aulas, horário das aulas, vestuário e calçado; f) intensidade da aula mantida entre três e quatro METs; g) idealização de um esquema padrão de aula. Assim, pode-se dizer que uma das maiores contribuições do projeto IMMA remete ao desenvolvimento de uma didática específica para prescrever exercícios físicos para pessoas idosas, o que tem feito do projeto um exemplo para muitos outros no Brasil afora.

A avaliação ampla do idoso é um processo multidimensional (geralmente interdisciplinar) destinado a formar um julgamento de valor sobre o status de saúde, as capacidades físicas, intelectuais e funcionais da pessoa idosa. O preceito de fazer passar por exame médico todos os candidatos a um programa de exercícios físicos mostra-se imprescindível quando se trata de idosos. As características marcantes da heterogeneidade da clientela-alvo exigem um exame médico funcional, além de uma avaliação antropométrica, como forma de fornecer indicadores do status de saúde e estado nutricional do idoso. ${ }^{15}$

Completando as rotinas que precedem a participação nas atividades, a pressão arterial de todos os idosos é aferida antes de cada aula e comparada com as informações sobre a preexistência de quadro de hipertensão arterial e o nível de normalidade obtido com o uso de medicamentos. De posse desses dados, o profissional de educação física tem condições de decidir sobre a participação ou não na aula e sobre o encaminhamento ao médico. 
Além disso, é importante tomar algumas precauções no caso de idosos portadores de diabetes, em função do risco da realização de atividades físicas com valores elevados ou demasiadamente reduzidos de glicemia. Após a aula, a glicose sanguínea volta a ser controlada, de maneira que se evitem episódios de hipoglicemia. Todas as rotinas preventivas são exibidas na lista de chamada aplicada pelos professores, onde, além do nome dos alunos, visualizam-se rapidamente os possíveis comprometimentos de cada aluno, de forma a auxiliar no processo decisório e facilitar a atenção individualizada, mesmo que a aula se dê em grupos. Como dito, procedimentos ligados a cuidados com o ambiente físico e o material para as aulas, horário das aulas, vestuário e calçado completam esses procedimentos, chegando até à decisão de suspensão das aulas em dias de temperatura muito elevada.

A questão da segurança nos exercícios físicos inclui também a questão da intensidade da aula, que deve ser mantida entre três e quatro METs, dadas as características e especificidades da população-alvo. Como forma de controle da intensidade das atividades, desenvolveu-se uma conjugação da escala de Borg de 10 pontos para a medida da sensação subjetiva de esforço com uma escala pictórica, de forma a superar as dificuldades acarretadas pelo baixo nível de escolaridade e possíveis deficits cognitivos dos idosos a quem o projeto atende.

Enfim, para melhor controlar a questão da segurança nos exercícios físicos e aprimorar o desempenho das aulas, foi idealizado um esquema padrão de aula. Defende-se a ideia de que as aulas de atividades físicas para idosos sejam elaboradas obedecendo a um esquema pedagogicamente predeterminado, ainda que suficientemente flexível para permitir adequações, e com base nas transformações que o envelhecimento não patológico acarreta. Com a adoção de um esquema, procura-se inibir "tentativas perigosas de originalidade". O esquema IMMA para aulas de ginástica/dança é composto de seis partes: 1) aquecimento, que emprega diferentes alongamentos e formas de andar, individualmente, em duplas ou em pequenos grupos, com variação de ritmo e envolvendo diferentes segmentos corporais; 2) flexibilidade e trabalho muscular geral, trabalho que visa melhorar a mobilidade articular (com os alunos em duplas ou pequenos grupos, geralmente sentados) e a força, com trabalho com cargas leves; 3) equilíbrio (estático e dinâmico), que objetiva o fortalecimento das estruturas muscular e articular, essenciais ao equilíbrio, com mudanças de posição; 4) movimentos de coordenação e imitação, incluindo reprodução de sequências de movimento, envolvendo dois ou mais segmentos corporais. Nesta parte, são ainda incluídos exercícios de reforço de um programa de reeducação postural (marcha, subida de escadas, reeducação respiratória, mudanças de decúbito etc.); 5) movimentos de deslocamento no espaço, com elementos da ginástica e da dança, seguindo ou não determinada música ou marcação rítmica, envolvendo elementos básicos como: gestos, giros e voltas simples, transferência de peso corporal em diferentes passos e formações; 6) relaxamento, procurando evitar o aumento das tensões musculares, por meio de movimentos lentos e suaves. Procura-se restabelecer o equilíbrio respiratório e circulatório. Inclui-se nesta parte a possibilidade de discussões sobre temas ligados à saúde (principalmente as atividades físicas) e ao exercício da cidadania de forma geral.

Evidentemente, um esquema padrão não é suficiente para garantir a qualidade do ensino, superar a inadequação das tentativas de adaptação, impedir a infantilização das propostas e levá-lo pelos caminhos de abordagens multiculturais. Sustenta-se no projeto IMMA a ideia de que não se devem fazer adaptações de desportos criados para jovens e adultos, e sim criar especificamente desportos e atividades para as pessoas idosas.

O projeto está passando por um momento de expansão, com aumento de sua estrutura física e humana, graças a investimentos conseguidos com agências de fomentos (CNPq e FAPERJ) e a uma parceria firmada com a Secretaria Especial de Envelhecimento Saudável e Qualidade de Vida (SESQV) da Prefeitura do Rio de Janeiro, que garantiu a estruturação de um centro de treinamento com equipamentos de última geração, bem como a contratação de profissionais qualificados. Isso permitiu o aumento do número de turmas e idosos atendidos e a inclusão de sessões individualizadas de treinamento aeróbio e de força muscular mais intensa. Hoje o projeto possui quatro turmas, atende 120 idosos e tem, em médio prazo, a capacidade de chegar a seis 
turmas, com 200 alunos.

\section{Academia da Terceira Idade (ATI)}

A Prefeitura do Rio de Janeiro, por intermédio de sua Secretaria Especial de Envelhecimento Saudável e Qualidade de Vida (SESQV), acredita que a qualidade de vida urbana está diretamente ligada a vários fatores que estão reunidos na infraestrutura, no desenvolvimento econômico-social e naqueles ligados à questão ambiental. Partindo desse pressuposto, a Prefeitura da Cidade do Rio de Janeiro, por meio da SESQV, criou em agosto de 2009 o projeto Academia da Terceira Idade (ATI). O programa consiste em promover a atividade física utilizando aparelhos de ginástica específicos para a prática de treino de força e aeróbio, preferencialmente para idosos, nos espaços públicos, em áreas como praças e parques da cidade do Rio de Janeiro.

Os objetivos das ATIs são: a) oportunizar aos idosos da cidade do Rio de Janeiro maior conscientização sobre a importância da prática da atividade física, contribuindo consequentemente para um envelhecimento mais saudável e com maior qualidade de vida; b) assegurar a participação dos idosos em ações profiláticas para a manutenção da saúde e impulsionar o conceito de saúde, estimulando a prática da atividade física como forma de prevenção; c) proporcionar ganhos de qualidade de vida, mediante a prática regular de atividade física, diminuindo os riscos para a aquisição de doenças crônico-degenerativas e consequentemente, a redução no risco de aquisição de patologias relacionadas a tal enfermidade; d) buscar a manutenção e/ou melhora da autonomia funcional, prevenção de quedas e a formação de redes sociais dos participantes.

As ATls são compostas por máquinas articuladas que não dispõem de regulagens para aumento progressivo da carga. A resistência imposta provém do peso dos segmentos corporais e das relações de alavanca contidas nos aparelhos. Cada ATI conta com duas equipes multidisciplinares, compostas de um profissional de educação física, um técnico em enfermagem e um apoio administrativo. Uma das equipes atua no período da manhã e outra no período da tarde. $\mathrm{O}$ público-alvo são os idosos (população acima dos 60 anos) e também adultos com idade superior a 40 anos, de forma a conscientizar este público sobre os benefícios que a atividade física pode proporcionar, buscando o envelhecimento ativo como alternativa de longevidade com qualidade.

Em junho de 2012, o Município do Rio de Janeiro, por meio da SESQV, firmou um protocolo de cooperação técnica com o Instituto de Educação Física e Desporto da UERJ, para implantar dentro de suas dependências uma ATI escola, a ATI-UERJ, tornando esta um Centro de Excelência Acadêmica e Laboratório Prático. A parceria tem como objetivo desenvolver estudos sobre o envelhecimento, oferecendo a ATI como local de estágio de campo e qualificação para os profissionais que trabalham nas ATIs da Prefeitura/SESQV distribuídas pela cidade, gerando indicadores de impacto no projeto ATI. Hoje, o projeto segue um planejamento organizado pelos coordenadores técnicos da Coordenadoria de Projetos e Estudos e Pesquisa (CPEP) da SESQV, em parceria com o LABSAU, de forma a orientar os professores sobre a metodologia e a conduta dos idosos do programa, mediante uma capacitação trimestral dos profissionais, com as diretrizes mais atuais no que se refere aos programas de atividade física para idosos.

As atividades do projeto ATI seguem uma metodologia específica para minimizar o problema da segurança na atividade física. O aluno, ao ingressar no programa, apresenta o atestado médico e responde a uma anamnese sobre fatores de risco para o desenvolvimento de doenças crônico-degenerativas. O técnico de enfermagem afere e anota diariamente a pressão arterial, de modo a identificar possíveis alterações.

A metodologia de aula segue a seguinte ordem: a) aferição da pressão arterial e chamada (10 min); b) exercícios de aquecimento (5 min); c) exercícios de alongamento (5 min), exercícios proprioceptivos ( $5 \mathrm{~min}$ ), treinamento em circuito (30 min), alongamento (5 min). O treinamento é realizado em circuito, com os alunos seguindo a ordem previamente estabelecida na rotina do programa. O programa tem a duração de uma hora e é realizado de segunda a sexta, nos horários das $7 \mathrm{~h}$ às $10 \mathrm{~h}$, no período da manhã, e das $16 \mathrm{~h}$ às $19 \mathrm{~h}$, no período da tarde (das 15h às $18 \mathrm{~h}$ na ATI-UERJ), sendo os idosos incentivados a utilizar os equipamentos, prioritariamente, nos horários em que a equipe está presente. Para maior integração dos alunos do projeto, são realizados eventos cultu- 
rais, como a comemoração de datas significativas para a comunidade. Tais eventos são considerados importantes para o processo de integração social da população adulta e idosa da cidade.

A mensuração do benefício gerado por este projeto ocorre de modo quantitativo, de acordo com o número de idosos inscritos e frequência mensal, controlada pela lista de chamada. Para somar-se a essa avaliação, a SESQV e o LABSAU, em 2013, planejam a realização de uma avaliação qualitativa, semestral, utilizando questionários de avaliação da qualidade de vida, como o WHOQOL -Old, instrumento transcultural, elaborado pela OMS para medir a qualidade de vida, e a bateria de testes de Fullerton, para aferir a aptidão física funcional da população idosa. Esses instrumentos permitirão a avaliação do impacto da prestação do serviço e de diferentes estruturas de atendimento social e saúde sobre a qualidade de vida dos idosos, especialmente na identificação das possíveis consequências das políticas implantadas e uma compreensão mais clara das áreas de investimento, para se obter ganhos mais significativos na qualidade de vida dessa população. ${ }^{39}$

A SESQV acredita que parcerias com instituições de ensino e pesquisa, como é o caso do LABSAU, podem trazer resultados no âmbito da academia, em publicação de pesquisas científicas e análise dos resultados, bem como no treinamento dos profissionais envolvidos, elevando a qualidade do atendimento e proporcionando à população idosa do Rio de Janeiro uma melhor qualidade de vida, com autonomia e independência.

\section{Conclusão}

O exercício físico sistematizado pode produzir diversos benefícios, tanto na esfera física quanto psicossocial, proporcionando uma melhor qualidade de vida ao idoso. No entanto, deve ser administrado de maneira correta, para maior segurança dessa população. O LABSAU, por meio de seus projetos e parcerias, tem se esforçado para contribuir para a produção de conhecimento e treinamento de recursos humanos no que tange às questões do exercício físico e envelhecimento. Além disso, oferece oportunidades de engajamento da pessoa idosa em programas regulares de atividades físicas de diversas naturezas, contribuindo com o desenvolvimento dessa área de interesse no estado do Rio de Janeiro.

\section{Referências}

1. Mathers CD, Sadana R, Salomon JA, Murray CJL, Lopez AD. Estimates of DALE for 191 countries: methods and results. (Global programme on evidence for health policy working paper No. 16). Geneva: World Health Organization; 2000. p. 1-19.

2. World Health Organization. Dept. of Noncommunicable Disease Prevention and Health Promotion. Active ageing: a policy framework. Geneva: World Health Organization; 2002.

3. Instituto Brasileiro de Geografia e Estatística (IBGE). Censo Demográfico 2010 - Características gerais da população, religião e pessoas com deficiência. Rio de Janeiro; 2010.

4. Farinatti PTV. Envelhecimento, promoção da saúde e exercício: bases teóricas e metodológicas. $1^{\mathrm{a}} \mathrm{ed}$. Barueri (SP): Manole; 2008. 512 p.

5. Islam MM, Takeshima N, Rogers ME, Yamauchi T, Koizumi D, Wang Y, et al. Decline of Functional Fitness in Free Living Japanese Older Adults. AJESS. 2005;2(1):7-9.

6. Eskurza I, Donato AJ, Moreau KL, Seals DR, Tanaka $\mathrm{H}$. Changes in maximal aerobic capacity with age in endurance-trained women: 7-yr follow-up. J Appl Physiol (1985). 2002;92(6):2303-8. http://dx.doi. org/10.1152/japplphysiol.01124.2001.

7. Deary IJ, Johnson W, Gow AJ, Pattie A, Brett CE, Bates TC, et al. Losing one's grip: a bivariate growth curve model of grip strength and nonverbal reasoning from age 79 to 87 years in the Lothian Birth Cohort 1921. J Gerontol B Psychol Sci Soc Sci. 2011;66(6):699-707. http://dx.doi.org/10.1093/geronb/ gbr059.

8. Aagaard P, Suetta C, Caserotti P, Magnusson SP, Kjaer M. Role of the nervous system in sarcopenia and muscle atrophy with aging: strength training as a countermeasure. Scand J Med Sci Sports. 2010;20(1):49-64. http://dx.doi.org/10.1111/j.16000838.2009.01084.x

9. Al Snih S, Raji MA, Peek MK, Ottenbacher KJ. Pain, lower-extremity muscle strength, and physical function among older Mexican Americans. Arch Phys Med Rehabil. 2005;86(7):1394-400. http://dx.doi. org/10.1016/j.apmr.2004.12.038.

10. Todd JA, Robinson RJ. Osteoporosis and exercise. Postgrad Med J. 2003;79(932):320-3. http://dx.doi. org/10.1136/pmj.79.932.320.

11. Luís CIFF. Influência da Nutrição no Envelhecimento: A Caminho da Longevidade [Monografia]. Porto: Universidade do Porto; 2010.

12. Paffenbarger RS Jr, Blair SN, Lee IM, Hyde RT. 
Measurement of physical activity to assess health effects in free-living populations. Med Sci Sports Exerc. 1993;25(1):60-70.

13. Furtado HL. Atividade física e envelhecimento. In: Brasil C, editor. Viver é melhor opção: Envelhecer... faz parte! Rio de Janeiro: Quartet; 2012. p. 183-203.

14. American College of Sports Medicine, Chodzko-Zajko WJ, Proctor DN, Fiatarone Singh MA, Minson CT, Nigg CR, et al. American College of Sports Medicine Position Stand. Exercise and physical activity for older adults. Med Sci Sports Exerc. 2009;41(7):1510-30.

15. American College of Sports Medicine. ACSM's guidelines for exercise testing and prescription. 8th ed. Philadelphia: Lippincott Williams \& Wilkins; c2010. p. 190-4.

16. Alves Junior ED. Envelhecimento e vida saudável. Rio de Janeiro: Apicuri; 2009. 312 p.

17. Stathokostas L, Jacob-Johnson S, Petrella RJ, Peterson DH. Longitudinal changes in aerobic power in older men and women. J Appl Physiol (1985). 2004;97(2):781-9. http://dx.doi.org/10.1152/ japplphysiol.00447.2003.

18. Ferreira MT. O papel da atividade física na composição corporal de idosos. Rev Bras Ciênc Saúde. 2003;1(1):46-52.

19. Shephard RJ. Envelhecimento, Atividade Física e Saúde. $1^{\text {a }}$ ed. São Paulo: Phorte; 2003. 496 p.

20. Macardle WD. Fisiologia do Exercício - Nutrição, Energia e Desempenho Humano. $7^{\mathrm{a}}$ ed. Rio de Janeiro: Guanabara Koogan; 2011. 1172 p.

21. Farinatti PT, Soares PP. Cardiac output and oxygen uptake relationship during physical effort in men and women over 60 years old. Eur J Appl Physiol. 2009;107(6):625-31. http://dx.doi.org/10.1007/ s00421-009-1162-y.

22. Vasan RS, Larson MG, Leip EP, Kannel WB, Levy D. Assessment of frequency of progression to hypertension in non-hypertensive participants in the Framingham Heart Study: a cohort study. Lancet. 2001;358(9294):1682-6. http://dx.doi.org/10.1016/ S0140-6736(01)06710-1.

23. Pescatello LS, Franklin BA, Fagard R, Farquhar WB, Kelley GA, Ray CA, et al. American College of Sports Medicine position stand. Exercise and hypertension. Med Sci Sports Exerc. 2004;36(3):533-53. Epub 2004/04/13.

24. Macaluso A, De Vito G. Muscle strength, power and adaptations to resistance training in older people. Eur J Appl Physiol. 2004;91(4):450-72. http://dx.doi. org/10.1007/s00421-003-0991-3. Epub 2003/11/26.

25. Latham NK, Bennett DA, Stretton CM, Anderson CS. Systematic review of progressive resistance strength training in older adults. J Gerontol A Biol Sci Med Sci.
2004;59(1):48-61. Epub 2004/01/14.

26. Roubenoff R, Hughes VA. Sarcopenia: current concepts. J Gerontol A Biol Sci Med Sci. 2000;55(12):M716-24. Epub 2000/12/29.

27. Hurley BF, Redmond RA, Pratley RE, Treuth MS, Rogers MA, Goldberg AP. Effects of strength training on muscle hypertrophy and muscle cell disruption in older men. Int J Sports Med. 1995;16(6):378-84. http:// dx.doi.org/10.1055/s-2007-973024. Epub 1995/08/01.

28. Evans W. Functional and metabolic consequences of sarcopenia. J Nutr. 1997;127(Suppl. 5):998S-1003S. Epub 1997/05/01.

29. Hagerman FC, Walsh SJ, Staron RS, Hikida RS, Gilders RM, Murray TF, et al. Effects of highintensity resistance training on untrained older men I, strength, cardiovascular, and metabolic responses. J Gerontol A Biol Sci Med Sci. 2000;55(7):B336-46. Epub 2000/07/18.

30. Kalapotharakos VI, Michalopoulou M, Godolias G, Tokmakidis SP, Malliou PV, Gourgoulis V. The effects of high and moderate-resistance training on muscle function in the elderly. J Aging Phys Act. 2004;12(2):131-43. Epub 2004/06/30.

31. Fiatarone MA, Marks EC, Ryan ND, Meredith CN, Lipsitz LA, Evans WJ. High-intensity strength training in nonagenarians. Effects on skeletal muscle. JAMA. 1990;263(22):3029-34. Epub 1990/06/13.

32. Moraes H, Deslandes A, Ferreira C, Pompeu FAMS, Ribeiro P, Laks J. O exercício físico no tratamento da depressão em idosos: revisão sistemática. Rev Psiquiatr Rio Gd Sul. 2007;29(1):70-9.

33. Carneiro RS, Falcone E, Clark C, Prette ZD, Prette AD. Qualidade de vida, apoio social e depressão em idosos: relação com habilidades sociais. Psicol Reflex Crit. 2007;20(2):229-37.

34. Mello MT, Boscolo RA, Esteves AM, Esteves AM, Tufik S. O exercício físico e os aspectos psicobiológicos. Rev Bras Med Esporte. 2005;11(3):203-7. http:// dx.doi.org/10.1590/S1517-86922005000300010.

35. Guimarães JMN, Caldas CP. A influência da atividade física nos quadros depressivos de pessoas idosas: uma revisão sistemática. Rev Bras Epidemiol. 2006;9(4):481-92. http://dx.doi.org/10.1590/S1415790X2006000400009.

36. Wiles NJ, Haase AM, Gallacher J, Lawlor DA, Lewis G. Physical activity and common mental disorder: results from the Caerphilly study. Am J Epidemiol. 2007;165(8):946-54. http://dx.doi.org/10.1093/aje/ kwk070. Epub 2007/02/03.

37. Barnes DE, Yaffe K, Satariano WA, Tager IB. A longitudinal study of cardiorespiratory fitness and cognitive function in healthy older adults. J Am Geriatr Soc. 2003;51(4):459-65. http://dx.doi.org/10.1046/ j.1532-5415.2003.51153.x. Epub 2003/03/27. 
38. Johnson W, Deary IJ, McGue M, Christensen K. Genetic and environmental links between cognitive and physical functions in old age. J Gerontol B Psychol Sci Soc Sci. 2009;64(1):65-72. http://dx.doi.

Recebido: 02/10/2013.

Revisado: 26/10/2013.

Aprovado: 06/12/2013. org/10.1093/geronb/gbn033. Epub 2009/02/12.

39. World Health Organization. Manual WHOQOL-OLD. Copenhagen: European Office; 2006. p. 61

\section{Nádia L. Silva}

Laboratório de Atividade Física e Promoção da Saúde. Instituto de Educação Física e Desportos. Universidade do Estado do Rio de Janeiro. Rio de Janeiro, RJ, Brasil.

\section{Cristiane Brasil}

Secretaria do Envelhecimento Saudável e Qualidade de Vida. Prefeitura da Cidade do Rio de Janeiro. Rio de Janeiro, RJ, Brasil.

\section{Hélio Furtado}

Prefeitura da Cidade do Rio de Janeiro. Secretaria do Envelhecimento Saudável e Qualidade de Vida. Rio de Janeiro, RJ, Brasil.

\section{João Costa}

Laboratório de Atividade Física e Promoção da Saúde. Instituto de Educação Física e Desportos. Universidade do Estado do Rio de Janeiro. Rio de Janeiro, RJ, Brasil.

\section{Paulo Farinatti}

Laboratório de Atividade Física e Promoção da Saúde. Instituto de Educação Física e Desportos. Universidade do Estado do Rio de Janeiro. Rio de Janeiro, RJ, Brasil. 